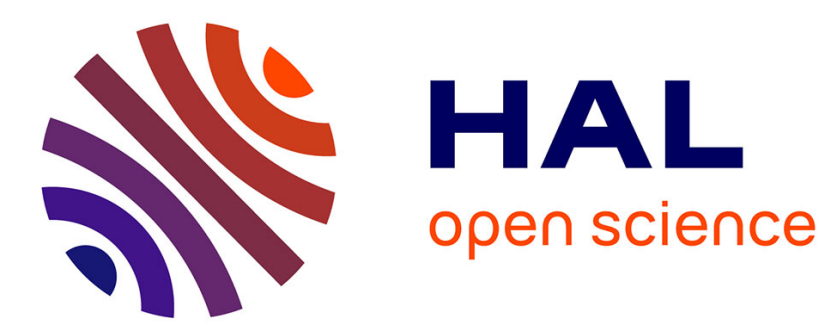

\title{
Face Recognition Based on Videos by Using Convex Hulls
}

Hakan Cevikalp, Hasan Serhan Yavuz, Bill Triggs

\section{To cite this version:}

Hakan Cevikalp, Hasan Serhan Yavuz, Bill Triggs. Face Recognition Based on Videos by Using Convex Hulls. IEEE Transactions on Circuits and Systems for Video Technology, 2020, 30 (12), pp.4481-4495. 10.1109/TCSVT.2019.2926165 . hal-03044541

\section{HAL Id: hal-03044541 \\ https://hal.science/hal-03044541}

Submitted on 7 Dec 2020

HAL is a multi-disciplinary open access archive for the deposit and dissemination of scientific research documents, whether they are published or not. The documents may come from teaching and research institutions in France or abroad, or from public or private research centers.
L'archive ouverte pluridisciplinaire HAL, est destinée au dépôt et à la diffusion de documents scientifiques de niveau recherche, publiés ou non, émanant des établissements d'enseignement et de recherche français ou étrangers, des laboratoires publics ou privés. 


\title{
Face Recognition Based on Videos by Using Convex Hulls
}

\author{
Hakan Cevikalp, Member, IEEE, Hasan Serhan Yavuz, Member, IEEE, and Bill Triggs, Member, IEEE
}

\begin{abstract}
A wide range of face appearance variations can be modeled by using set based recognition approaches effectively, but computational complexity of current methods is highly dependent on the set and class sizes. This paper introduces new video based classification methods designed for reducing the required disk space of data samples and speed up the testing process in large-scale face recognition systems. In the proposed method, image sets collected from videos are approximated with kernelized convex hulls and it was shown that it is sufficient to use only the samples that participate in shaping the image set boundaries in this setting. The kernelized Support Vector Data Description (SVDD) is used to extract those important samples that form the image set boundaries. Moreover, we show that these kernelized hypersphere models can also be used to approximate image sets for classification purposes. Then, we propose a binary hierarchical decision tree approach to improve the speed of the classification system even more. Lastly, we introduce a new video database that includes 285 people with 8 videos of each person since the most popular video data sets used for set based recognition methods include either a few people, or small number of videos per person. The experimental results on varying sized databases show that the proposed methods greatly improve the testing times of the classification system (we obtained speed-ups to a factor of 20) without a significant drop in accuracies.
\end{abstract}

Index Terms-Face Recognition, Image Set Classification, Affine Hulls, Convex Hulls, Support Vector Data Description, Binary Hierarchical Tree.

\section{INTRODUCTION}

$\mathrm{F}$ ACE recognition systems find their applications in various fields. Rather than typically being used in security systems, wide use of video or cell phone cameras have led to identifying people in daily life such as tagging a friend on social media or searching for some people among numerous videos etc.. In general, face recognition process can involve 1) single image based or 2) video (collection of a set of images) based classification tasks. For set based face recognition, both gallery and query sets are given in terms of sets of images rather than a single image. Images can be collected from video frames as well as from multiple unordered observations. The classification system must return the individual whose gallery set is the most similar to the given query set. Set based methods usually perform better than the methods using single images since image sets include variability of the individual's appearance. These methods are also more practical owing to the fact that they usually do not require any cooperation from persons. However, despite these advantages, traditional classifiers such as Support Vector Machines (SVMs), classification trees, $k$-nearest neighbor $(k-\mathrm{NN})$ etc. cannot be used directly, which can be considered as a major limitation of the set based methods.

Based on the set model representation types, existing set based methods can be roughly divided into two categories, parametric and non-parametric methods. Probability distributions such as Gaussians or mixture of them were used to model image sets in parametric methods such as $[1,2]$, and the similarity (or dissimilarity) is measured by using the distribution divergences. However, these methods do not perform well in cases where the test sets have only weak statistical relationships to the training ones as

- H. Cevikalp and H.S. Yavuz are with the Department of Electrical and Electronics Engineering, Eskisehir Osmangazi University, 26480, Turkey. B. Triggs is with Laboratoire Jean Kuntzmann, Grenoble, France.

E-mail: see http://mlcv.ogu.edu.tr/contacts.html noted in $[3,4]$. Nonparametric methods, on the other hand, use different models (e.g., linear or affine subspaces or some different combinations of these subspaces) to approximate image sets, and different metrics have been proposed for set-to-set distances based on the type of utilized models.

As a pioneering work for nonparametric models, Yamaguchi et al. [5] used linear subspaces to approximate image sets, and canonical angles between subspaces are used to measure the distance between them. A basic limitation of linear subspace methods is that the linear subspace angles do not provide strong information about the locations of the samples (affine subspaces can better approximate class regions compared to the linear ones). Another way of dealing with image set based classification is to consider each sample as a point in a Grassmannian manifold. Hamm and Lee [6] used Grassmannian discriminant analysis on fixed dimensional linear subspaces. Wang and Shi [7] proposed kernel Grassmannian distances to compare image sets. Harandi et al. [8] introduced a graph embedding framework which uses within-class and between-class similarity graphs to characterize intra-class variance and inter-class separability. More recently, manifolds of symmetric positive definite matrices are used to model images sets and the similarities between these manifolds are computed by using different Riemannian metrics such as Affine-Invariant metric or Log-Euclidean metric $[9,10]$. We introduced affine and convex hulls to approximate image sets in [3], and geometric distances between these models are used to measure the similarity. Different variants of affine and convex hulls have been proposed in $[11,12]$ after introduction of affine and convex hulls. Among these, Sparse Approximated Nearest Points (SANP) of [11] enforces the sparsity of samples used for affine hull combination. SANPs of two sets are first approximated by using affine hulls of the sets, then they are sparsely approximated from the set images while simultaneously searching for the closest points between sets. Despite its good accuracies, this method requires setting three 
parameters in addition to the affine hull parameters. Therefore, it is complex. Furthermore, the method is also slow since it requires solving an optimization problem which requires minimization of L1 norm of some vectors. Similarly, [12] used regularized affine hull models that includes minimization of L2-norms of affine hull combination coefficients during computing the smallest distances between image sets. Wang et al. [13] proposed a method to learn more compact and discriminative affine hulls when the affine hulls of different classes overlap. More recently, new extensions [14, 15] of these methods used the so-called collaborative representations for affine and convex hull models. In contrast to the traditional methods using an independent affine and convex hull for each image set, these methods approximate all gallery sets by using a single affine or convex hull and the query set is labeled by using the reconstruction residuals computed from only individual gallery sets. However, as demonstrated in the experiments, these methods mostly fail for large-scale applications. Other representative methods using sparse models in image set based recognition can be found in $[16,17,18]$. Most of the aforementioned methods have kernelized versions that can be used to approximate nonlinear face models.

In addition to these, there are some methods that build nonlinear approximations of the manifold of face appearances by approximating local regions with linear models. For instance, local structures are found by using hierarchical clustering in [19], and each local region is approximated with a linear subspace. Wang et al. [4] use nearest neighbor clustering to find the local structures in manifold-to-manifold distance (MMD) method. This method was extended in a way that the between-manifold distances are improved in [20]. Spectral clustering was used to find the local structures in [3], and each local structure is modeled with affine subspaces. Hadid and Pietikainen [21] use $k$-means clustering to find local structures and model each local structure with the cluster center. More recently, Hayat et al. [22,23] proposed a deep learning framework to estimate the nonlinear geometric structure of the image sets. They trained an Adaptive Deep Network Template for each image set to learn the class-specific models and then the query set is classified based on the minimum reconstruction error computed by using those pre-learnt class-specific models.

Lastly, there are also some related face verification and identification methods using face image sets [24,25,26,27]. For example, Liu et al. [27] use multitask joint sparse representation algorithm for video-based verification. Liu et al. [24] and Rao et al. [26] use deep neural network based methods to find high quality discriminative face image frames within the image sets to improve the accuracy and speed of the face identification systems. In a similar manner, Yang et al. [25] combine a CNN network and an aggregation module to create a discriminative image set model by using high-quality image frames for video based face recognition.

Our Contributions: In the aforementioned studies, experimental evaluations have been performed on small sized video databases in general. In this paper, we focus on large-scale face recognition applications using image sets and discuss the main challenges that may be encountered in dealing with large-scale data. Then, we examine the suitability of existing methods in large-scale settings. Finally, we propose an efficient method for large-scale set based face recognition. In the proposed method, the most essential samples in image sets were extracted to reduce the image set samples, and the classification is accomplished by using the reduced image sets. To reduce image sets, we use the Support Vector Data Description (SVDD) method of [28] which returns a compact kernelized hypersphere that best fits the image set samples. In addition, we show how to use the kernelized hypersphere models for set based recognition and introduce a binary hierarchical tree approach to improve the speed of classification stage even more. We also collected a new video data set called ESOGU-285 Face Videos since the most popular video data sets used for set based recognition methods are not large-scale and they include only few person classes. There are 2280 videos belonging to 285 individuals in our dataset. The total number of frames is about 764K. Although this data set cannot be considered large-scale data, it is typically larger than the valid conventional datasets and it was still sufficient to show that many face recognition methods using image sets have serious drawbacks related to computational complexity or representation of image sets. Preliminary versions of this paper have appeared in [3,29]. This paper extends our previous work with (1) a more detailed analysis of the recent related work on set based face recognition; (2) a more detailed description of the linear/kernelized affine/convex hull distances; (3) introduction of a novel binary hierarchical approach to speedup the classification stage, and (4) more experiments on both small and moderate sized face video datasets.

The remainder of the paper is organized as follows: Section 2 reviews affine/convex hull based face recognition methods. We discuss challenges of large-scale set based face recognition in Section 3. Section 4 introduces the proposed revisions that will make large-scale image set based recognition feasible using kernelized convex hull models. Section 5 summarizes experimental results. Lastly, our conclusions are presented in Section 6.

\section{Affine/Convex Hull Approximations}

Set based classification methods include two important steps: 1) finding models to represent image sets; 2) defining suitable distance measures between the models. Depending on the representation types, different distance metrics are used to compute the distances between sets. Among these, nonparametric set based representations have been reported to produce more promising results than the parametric ones. The central idea of the current nonparametric methods is to represent the image sets by some geometric structure either in terms of some restricted geometric surfaces or directly in terms of spanning subspaces. Among the set representation methods, affine hull or convex hull based representations have some important attractive properties. In these methods, we can fit an independent model for each individual; finding distances between the models is straightforward due to convexity; robust fitting methods can be easily adopted to cope with outliers and the models are suitable to be used with the kernel trick so that the advantages of nonlinear structures can be utilized in the classification.

Let the face image samples be $\mathbf{x}_{c i} \in \mathbb{R}^{d}$ where $c=1, \ldots, C$ indexes the $C$ image sets (individuals) and $i=1, \ldots, n_{c}$ indexes the $n_{c}$ samples of image set $c$. [3] approximates image sets with a convex model (either an affine or convex hull) and test image set is assigned to the class with the closest gallery set.

The closest distance between two convex sets $H$ and $H^{\prime}$ is the minimum of the distances between any point in $H$ and any point in $H^{\prime}$ :

$$
D\left(H, H^{\prime}\right)=\underset{\mathbf{x} \in H, \mathbf{y} \in H^{\prime}}{\arg \min }\|\mathbf{x}-\mathbf{y}\| .
$$


To find this distance, parametric forms for the points in $H$ and $H^{\prime}$ must be introduced. Then, inter-points distances can be minimized using mathematical programming.

\subsection{Affine Hull Models}

In this method, image sets are approximated by the affine hulls (affine subspaces that can be seen as shifted linear subspaces) of their training samples:

$$
H_{c}^{\mathrm{aff}}=\left\{\mathbf{x}=\sum_{k=1}^{n_{c}} \alpha_{c k} \mathbf{x}_{c k} \mid \sum_{k=1}^{n_{c}} \alpha_{c k}=1\right\}, c=1, \ldots, C .
$$

The affine model basically regards any affine combination of an individual's feature sample vectors as a valid face feature sample for that person. This typically gives a very loose approximation to the data, since affine model does not specify where the class samples lie within the affine subspaces. There are basically two ways to find the distances between affine hulls: Subspace based solution and quadratic programming (QP) based solution.

Distance Computation by Using Subspace Formulation: In this approach, we start by selecting a reference point on the affine hull. This reference point can be one of the face image samples of a set or it can be the mean face image of the set. Let the reference point be denoted as $\boldsymbol{\mu}_{c}$. Then, the affine model of set $c$ in terms of this point is written as:

$$
H_{c}^{\text {aff }}=\left\{\mathbf{x}=\boldsymbol{\mu}_{c}+\mathbf{U}_{c} \mathbf{v}_{c} \mid \mathbf{v}_{c} \in \mathbb{R}^{l}\right\}
$$

Here, $\mathbf{U}_{c}$ is an orthonormal basis for the directions spanned by the affine subspace, $\mathbf{v}_{c}$ is a vector of free parameters that determines the coordinates for the points within the subspace, expressed with respect to the basis $\mathbf{U}_{c}$, and $l$ is the number of the basis vectors. Numerically, $\mathbf{U}_{c}$ is obtained by applying the thin Singular Value Decomposition to $\left[\mathbf{x}_{c 1}-\boldsymbol{\mu}_{c}, \ldots, \mathbf{x}_{c n_{c}}-\boldsymbol{\mu}_{c}\right]$. We usually discard the directions corresponding to very small singular values to remove noisy dimensions within data. The effective dimension of $\mathbf{U}_{c}$ and the hull is the number of significantly non-zero singular values.

Given two non-intersecting affine hulls $\left\{\mathbf{U}_{c} \mathbf{v}_{c}+\boldsymbol{\mu}_{c}\right\}$ and $\left\{\mathbf{U}_{c^{\prime}} \mathbf{v}_{c^{\prime}}+\boldsymbol{\mu}_{c^{\prime}}\right\}$, the closest points on them that gives the distance between the affine hulls can be found by solving the following optimization problem

$$
\underset{\mathbf{v}_{c}, \mathbf{v}_{c^{\prime}}}{\arg \min }\left\|\left(\mathbf{U}_{c} \mathbf{v}_{c}+\boldsymbol{\mu}_{c}\right)-\left(\mathbf{U}_{c^{\prime}} \mathbf{v}_{c^{\prime}}+\boldsymbol{\mu}_{c^{\prime}}\right)\right\|^{2} .
$$

Defining $\mathbf{U} \equiv\left(\begin{array}{ll}\mathbf{U}_{c} & -\mathbf{U}_{c^{\prime}}\end{array}\right)$ and $\mathbf{v} \equiv\left(\begin{array}{c}\mathbf{v}_{c} \\ \mathbf{v}_{c^{\prime}}\end{array}\right)$, this can be written as a standard least squares problem

$$
\underset{\mathbf{v}}{\arg \min }\left\|\mathbf{U} \mathbf{v}-\left(\boldsymbol{\mu}_{c^{\prime}}-\boldsymbol{\mu}_{c}\right)\right\|^{2},
$$

whose solution is $\mathbf{v}=\left(\mathbf{U}^{\top} \mathbf{U}\right)^{-1} \mathbf{U}^{\top}\left(\boldsymbol{\mu}_{c^{\prime}}-\boldsymbol{\mu}_{c}\right)$. So, the distance between the hulls becomes

$$
D\left(H_{c}^{\mathrm{aff}}, H_{c^{\prime}}^{\mathrm{aff}}\right)=\left\|(\mathbf{I}-\mathbf{P})\left(\boldsymbol{\mu}_{c}-\boldsymbol{\mu}_{c^{\prime}}\right)\right\|
$$

where $\mathbf{P}=\mathbf{U}\left(\mathbf{U}^{\top} \mathbf{U}\right)^{-1} \mathbf{U}^{\top}$ is the orthogonal projection matrix of the joint span of the directions contained in the two subspaces, and $(\mathbf{I}-\mathbf{P})$ is the projection matrix of the orthogonal complement of this span. The final set to set distance measure given in (6) is called the affine hull based image sets distance.
Distance Computation by Using Quadratic Programming (QP): Suppose $\mathbf{X}_{c}$ is a matrix whose columns are the feature vectors of set $c$ and $\boldsymbol{\alpha}_{c}$ is a vector containing the corresponding $\alpha_{c k}$ coefficients. Then, the affine hull given in (2) can be written in the form $\left\{\mathbf{x}=\mathbf{X}_{c} \boldsymbol{\alpha}_{c}\right\}$ with the same constraint. In this case, the distance between two affine hulls can be found by solving the following constrained convex quadratic optimization problem

$$
\begin{aligned}
& \left(\boldsymbol{\alpha}_{c}^{*}, \boldsymbol{\alpha}_{c^{\prime}}^{*}\right)=\underset{\boldsymbol{\alpha}_{c}, \boldsymbol{\alpha}_{c^{\prime}}}{\arg \min }\left\|\mathbf{X}_{c} \boldsymbol{\alpha}_{c}-\mathbf{X}_{c^{\prime}} \boldsymbol{\alpha}_{c^{\prime}}\right\|^{2} \\
& \text { s.t. } \quad \sum_{k=1}^{n_{c}} \alpha_{c k}=1=\sum_{k^{\prime}=1}^{n_{c^{\prime}}} \alpha_{c^{\prime} k^{\prime}} .
\end{aligned}
$$

The QP formulation is useful especially when the affine hulls of persons lightly intersect (i.e., the hulls intersect because of only a few samples). Note that the distance computation between affine hulls may fail if several gallery hulls intersect the given test one, since in this case the test class will have zero distance for more than one gallery class. This can occur under the existence of outliers (incorrect or very poor images) in any of the image sets. This problem can be solved by using a more robust hull fitting procedure. To prevent the intersection of the gallery sets, one can use a reduced representation to control the looseness of the model. The reduced representation can be formed by introducing lower and upper bounds $L, U$ on the allowable $\alpha$ coefficients in (2) as given in the following

$$
H_{c}^{\mathrm{raff}}=\left\{\mathbf{x}=\sum_{k=1}^{n_{c}} \alpha_{c k} \mathbf{x}_{c k} \mid \sum_{k=1}^{n_{c}} \alpha_{c k}=1, L \leq \alpha_{c k} \leq U\right\} .
$$

In the affine hull case, the bounds become $(L, U)=(-\infty, \infty)$. For the convex hull case, $L=0$ and $U \leq 1$. If $L=0$ and $U<1$, several samples need to be active to ensure $\sum_{k} \alpha_{c k}=1$, giving a reduced convex approximation that lies inside the convex hull of the samples. Similarly, the bounds $-\infty<L<0, U \geq 1$, results in a convex region which is larger than the convex hull, but smaller than the affine one.

The points of $H_{c}^{\text {raff }}$ can be written in a more compact form, $\left\{\mathbf{x}=\mathbf{X}_{c} \boldsymbol{\alpha}_{c}\right\}$, as before. $H_{c}^{\text {raff }}$ is convex, because any convex sum of its points, i.e., of $\boldsymbol{\alpha}_{c}$ vectors satisfying the sum 1 and $L, U$ constraints still satisfies these constraints. We apply the same $L, U$ constraints to each $\alpha_{c k}$ coefficient for simplicity.

The distance between two reduced hulls can be found by solving the following constrained convex optimization problem

$$
\begin{aligned}
& \left(\boldsymbol{\alpha}_{c}^{*}, \boldsymbol{\alpha}_{c^{\prime}}^{*}\right)=\underset{\boldsymbol{\alpha}_{c}, \boldsymbol{\alpha}_{c^{\prime}}}{\arg \min }\left\|\mathbf{X}_{c} \boldsymbol{\alpha}_{c}-\mathbf{X}_{c^{\prime}} \boldsymbol{\alpha}_{c^{\prime}}\right\|^{2} \\
& \text { s.t. } \quad \sum_{k=1}^{n_{c}} \alpha_{c k}=1=\sum_{k^{\prime}=1}^{n_{c^{\prime}}} \alpha_{c^{\prime} k^{\prime}}, \quad L \leq \alpha_{c k}, \alpha_{c^{\prime} k^{\prime}} \leq U
\end{aligned}
$$

and taking $D\left(H_{c}^{\text {raff }}, H_{c^{\prime}}^{\text {raff }}\right)=\left\|\mathbf{X}_{c} \boldsymbol{\alpha}_{c}^{*}-\mathbf{X}_{c^{\prime}} \boldsymbol{\alpha}_{c^{\prime}}^{*}\right\|$.

The feature vectors of set image samples $\mathbf{x}_{c k}$ only appear in the quadratic term of (9); therefore, the method can be kernelized by rewriting the quadratic in terms of dot products $\mathbf{x}_{c k}^{\top} \mathbf{x}_{c^{\prime} k^{\prime}}$ and replacing these with kernel evaluations $k\left(\mathbf{x}_{c k}, \mathbf{x}_{c^{\prime} k^{\prime}}\right)$. All gallery and test samples retain in their corresponding models, thus the coefficients are not sparse except for convex models. However, the distance computation is not very complex because each individual is fitted separately. So, one has to solve smaller sized optimization problems. 


\subsection{Convex Hull Models}

The convex hull of a set is defined as the smallest convex set containing its samples. When the full affine hull representation given in (2) is restricted for only positive $\alpha_{c k}$ coefficients, it represents the minimal convex set, i.e., the convex hull of the set.

$$
H_{c}^{\text {convex }}=\left\{\mathbf{x}=\sum_{k=1}^{n_{c}} \alpha_{c k} \mathbf{x}_{c k} \mid \sum_{k=1}^{n_{c}} \alpha_{c k}=1, \alpha_{c k} \geq 0\right\}
$$

Convex hull representation of sets is much tighter than the affine approximation. However, it can underestimate the true extent of the underlying class particularly for small numbers of samples in high dimensions.

By setting $L=0$ and no $U$ constraint in (7), the distances between the convex hulls can be computed. It should be noted that linear SVM also uses convex hulls to approximate binary classes, thus one can find the distances between convex hulls by training an SVM classifier that discriminates the test set from the given gallery one at run time. SVM returns the best separating hyperplane parameters, $(\mathbf{w}, b)$, and the distance is equal to $2 /\|\mathbf{w}\|$ where $\mathbf{w}$ is the normal of the separating hyperplane. To cope with outliers, we can set $U<1$ to obtain a more restrictive approximation. This problem is similar to the soft-margin SVM and the $\nu$-SVM [30].

As in the affine case, we can also use the kernel trick to extend the method to the nonlinear case. Let $\phi($.$) be the implicit$ feature space embedding and $k\left(\mathbf{x}_{i}, \mathbf{x}_{j}\right)=\left\langle\phi\left(\mathbf{x}_{i}\right), \phi\left(\mathbf{x}_{j}\right)\right\rangle$ be the corresponding kernel function, where $\langle$.$\rangle denotes the feature space$ inner product. A kernelized convex hull of samples $\mathbf{x}_{c k}$ can be defined as

$$
H_{c}^{\mathrm{kconvex}}=\left\{\begin{array}{c}
\phi(\mathbf{x})=\sum_{k=1}^{n_{c}} \alpha_{c k} \phi\left(\mathbf{x}_{c k}\right) \text { s.t. } \\
\sum_{k=1}^{n_{c}} \alpha_{c k}=1,0 \leq \alpha_{c k} \leq 1 .
\end{array}\right\}
$$

As in the linear case, if we set the upper bound on $U$ to values smaller than 1, several samples need to be activated to ensure $\sum_{k=1}^{n_{c}} \alpha_{c k}=1$, giving a more compact convex approximation that lies strictly inside the kernelized convex hull of the samples.

The distance between two kernelized convex hulls can be found by solving the following convex quadratic programming (QP) problem

$$
\begin{aligned}
\underset{\boldsymbol{\alpha}_{c}, \boldsymbol{\alpha}_{c^{\prime}}}{\arg \min } & \left\|\Phi\left(\mathbf{X}_{c}\right) \boldsymbol{\alpha}_{c}-\Phi\left(\mathbf{X}_{c^{\prime}}\right) \boldsymbol{\alpha}_{c^{\prime}}\right\|^{2} \\
\text { s.t. } & \sum_{k=1}^{n_{c}} \alpha_{c k}=\sum_{k^{\prime}=1}^{n_{c^{\prime}}} \alpha_{c^{\prime} k^{\prime}}=1, \quad 0 \leq \alpha_{c k}, \alpha_{c^{\prime} k^{\prime}} \leq U,
\end{aligned}
$$

where $\Phi\left(\mathbf{X}_{c}\right)=\left[\phi\left(\mathbf{x}_{c 1}\right), \ldots, \phi\left(\mathbf{x}_{c n_{c}}\right)\right]$ represents the matrix whose columns are the implicitly mapped samples of set $c$, and $\boldsymbol{\alpha}_{c}$ is a vector containing the corresponding $\alpha_{c k}$ coefficients. The objective function of (12) can be written as $\boldsymbol{\alpha}^{\top} \mathbf{K} \boldsymbol{\alpha}$ by setting $\Phi(\mathbf{X})=\left[\Phi\left(\mathbf{X}_{c}\right)-\Phi\left(\mathbf{X}_{c^{\prime}}\right)\right]$ and $\boldsymbol{\alpha} \equiv\left(\begin{array}{c}\boldsymbol{\alpha}_{c} \\ \boldsymbol{\alpha}_{c^{\prime}}\end{array}\right)$, where $\mathbf{K}=\Phi(\mathbf{X})^{\top} \Phi(\mathbf{X})$ is a positive semi-definite matrix. Therefore, the quadratic optimization problem is convex and there exists a global minimum.

\section{Challenges for large-Scale face Recognition Based on Image Sets}

In contrast to face recognition methods using single images, methods using image sets need a larger space to store all data since even short videos include hundreds of frames. Therefore, the first major challenge for large-scale applications will be related to saving all data in a computer. To overcome this limitation, the best strategy is to reduce the original image data without sacrificing recognition performance much. Reducing image data through random selection is not a good strategy since this significantly decreases the recognition performance as reported in $[17,18,11]$. Another challenge will be related to choosing a good model for approximating image sets. When image sets are constructed from videos, images include different poses of individuals such as frontal poses, left/rigt profiles, and poses between these. Consequently, the resulting image sets form a nonlinear manifold which is mostly locally linear. Currently, the methods approximating these non-linear image set manifolds with single linear/affine subspaces yield good accuracies on small image set datasets, but the accuracies will drop as the size of the datasets is increased. This performance drop occurs because these models will introduce large overlapping regions between image sets as illustrated in Fig. 1 since they use very loose models to approximate real image set regions. Therefore, methods using nonlinear approximations such as kernelized affine/convex hulls or methods building nonlinear manifolds by combining locally linear patches will perform well for large-scale applications. The last challenge will be the realtime performance of the recognition system. An efficient system must find the individual who is the most similar to the test person in reasonable time among thousands of people.

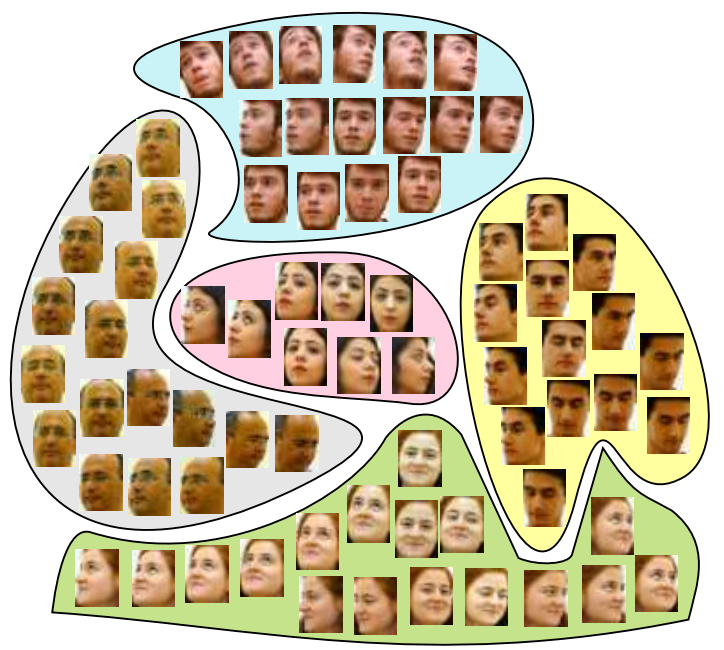

Fig. 1. Affine and convex hulls may over-estimate the true class regions, which causes large overlapping regions in large-scale applications. All images in each class span entire 2D plane for affine hull models in this example, thus it is impossible to separate image sets by using affine hulls. Convex hulls are more tight models compared to affine hulls, but most neighboring image sets have overlaps as illustrated in the example. Therefore, it is only possible to separate the furthest image sets for convex hull models.

We also would like to point out that the methods using joint or collaborative representations will be impractical and give inferior results for large-scale applications as illustrated in Fig. 2 although they achieve very high accuracies for small datasets. In these methods, a single affine/convex hull is used to approximate the entire gallery image sets, and a query set is classified based on the minimum length of reconstruction residuals that are computed by using individual gallery sets. If there are a few image sets in the gallery, one can approximate all image sets by using a single convex hull and compute the distance from the convex hull of the query to the combined convex hull of the gallery 
sets as shown in Fig. 2 (a). However, as the number of people is increased, the gallery images fill in the input space and the query set becomes surrounded by gallery sets and it lies in the combined convex hull of the gallery as illustrated in Fig. 2 (b). Consequently, the distances become zero and the coefficients that will be used for computing residuals become almost random, which in turn degrades the recognition accuracy. The situation is more severe for affine hulls since three independent images span entire 2-dimensional space given in Fig. 2. For some collaborative representation based methods as in [14], we should also expect computational difficulties since one has to take inverse of large matrices whose size depends on the total number of images in the gallery. For large-scale settings, it is even impractical to fit these matrices into the memory, not to mention the difficulty of taking their inverses. Our experiments at the end confirm this fact.

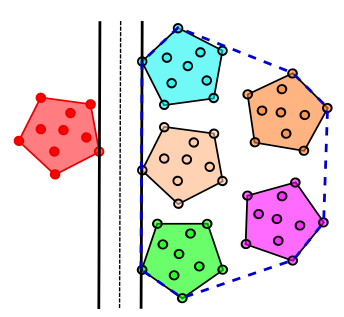

(a)

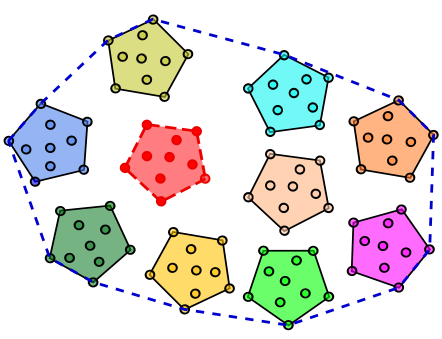

(b)
Fig. 2. An illustration for comparing small and large-scale scenarios for set based recognition: In (a), the number of the image sets belonging to different people in gallery is small; consequently, we can model all gallery sets as a single convex hull and find the distance from the convex hull of the query set to this joint hull. However, when the number of people is increased, the query sets will typically lie inside the joint convex hull of gallery sets as illustrated in (b). For such cases, the distances will become zero and the coefficients which will be used for computing residuals will be meaningless. This will cause the collaborative model classifier to fail.

\section{Proposed Method}

As stated before, only kernelized methods or methods that build nonlinear face manifolds by combining linear models can give good performance in large scale set based face recognition. However, if the number of classes is increased, the computational costs of all kernelized methods dramatically increase. Therefore, most of the methods reporting high recognition rates are not applicable because of impractical memory considerations due to large sized matrix inversion or the need of long time for convergence in optimization procedures. To ameliorate this problem, we propose a novel method to represent image sets with a reduced amount of samples without sacrificing recognition accuracy when kernelized convex hulls are used to approximate image sets. This approach both reduces the required disk space to save image sets and improves the testing time since less number of image set samples is used during computation of the distances between image sets.

\subsection{Reduced Convex Hull Models and Computing Dis- tances Between Them}

Convex hull modeling results in tighter models compared to affine hull modeling, so we used kernelized convex hulls for large scale applications in the proposed method. As mentioned earlier, finding the distances between two kernelized convex hulls is closely related to the SVM classifier solution since SVM also uses convex hull modeling to approximate classes. It should be noted that SVM classifiers in general return solutions which can be determined by using only the support vectors. Support vectors are the samples which lie nearest to the decision boundaries and all other samples do not contribute to the returned solution. The main objective of support vector machines training is to determine these essential points to build the classifier. We can adopt this idea for convex sets. The geometric distance between two arbitrary convex sets is determined based on the closest points between their boundaries. If we can define the boundaries of the convex regions with a few essential data points in the feature space, then we can calculate the distances by using only those points. This representation brings two important advantages: 1) there is no need to store the irrelevant data which reduces the required disk storage space, 2) testing speed increases since the new problem requires the solution of a smaller sized quadratic programming problem.

Finding Essential Image Set Samples: We can use kernelized one-class classifier methodologies presented in [28,31] to determine the essential image set samples which shape the convex hull boundaries. Considering the geometrical intuitions behind these methods, the SVDD method of Tax and Duin [28] is more suitable to use in our problem. SVDD method basically finds a compact bounding hypersphere inscribing the data and it is simply characterized by a center and radius. Let $\left\{\mathbf{x}_{k} \in \mathbb{R}^{d} \mid k=1, \ldots, n\right\}$ be any point set, and let us denote the radius of the bounding hypersphere of the point set with $r$ and it's center vector with $\mathbf{s}$. These parameters can be easily calculated by solving the following QP problem:

$$
\begin{aligned}
\underset{\mathbf{s}, r \geq 0, \boldsymbol{\xi} \geq 0}{\arg \min } & \left(r^{2}+\gamma \sum_{k} \xi_{k}\right) \\
\text { s.t. } & \left\|\mathbf{x}_{k}-\mathbf{s}\right\|^{2} \leq r^{2}+\xi_{k}, \quad k=1, \ldots, n,
\end{aligned}
$$

or its dual

$$
\begin{aligned}
\underset{\boldsymbol{\alpha}}{\arg \min } & \left(\sum_{k, l} \alpha_{k} \alpha_{l}\left\langle\mathbf{x}_{k}, \mathbf{x}_{l}\right\rangle-\sum_{k} \alpha_{k}\left\|\mathbf{x}_{k}\right\|^{2}\right) \\
\text { s.t. } & \sum_{k} \alpha_{k}=1, \quad \forall k 0 \leq \alpha_{k} \leq \gamma .
\end{aligned}
$$

Here, the $\alpha_{k}$ are Lagrange multipliers and $\gamma \in[1 / n, 1]$ is the ceiling parameter that can be tuned to reduce the influence of outliers. The resulting objective function is convex with a global minimum. Replacing the inner product terms $\left\langle\mathbf{x}_{k}, \mathbf{x}_{l}\right\rangle$ in the above equation with the kernelized functions $k\left(\mathbf{x}_{k}, \mathbf{x}_{l}\right)$ results in a kernelized version of the method. The dual formulation of the kernelized case typically yields a sparse solution in terms of the support vectors. Samples that correspond to the nonzero Lagrange multipliers are the support vectors and they can be used as the essential points which form the convex hull boundaries as illustrated on a toy data given in Fig. 3. If the ceiling parameter is set to small values, the algorithm will typically return more support vectors since more nonzero coefficients are needed to be active to ensure $\sum_{k=1}^{n} \alpha_{k}=1$, whereas the algorithm will return less support vectors for larger values of the ceiling parameter.

In the proposed method, we solve the quadratic problem in (14) for each image set in the gallery offline as the training phase and save only the essential points for each set. In testing phase, we run the same algorithm for the query set to determine the essential points of it and then we simply calculate the convex hull distances 


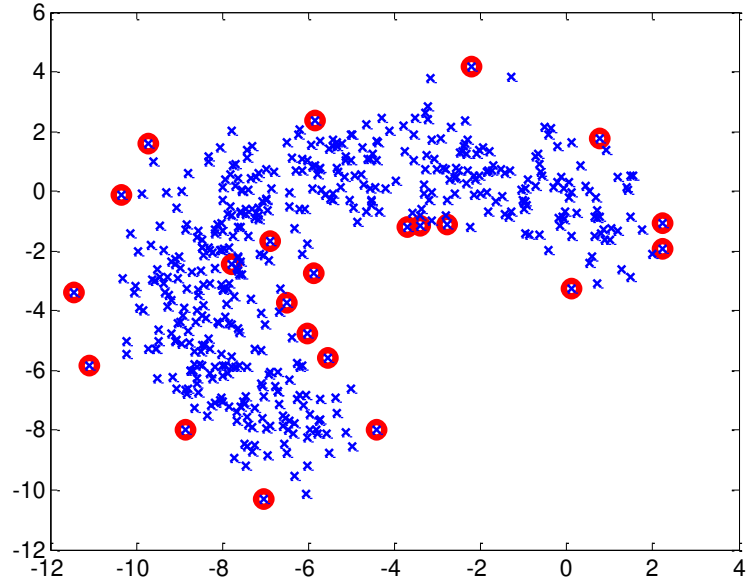

Fig. 3. Nonlinearly distributed toy data (shown with blue crosses) and the support vectors (shown with red circles around the data samples) returned by SVDD using a Gaussian kernel. Support vectors lie close to the object boundaries when the Gaussian kernel width is set properly.

between the query set and gallery sets. Here, set-to-set distances are computed by using only the essential points which constitute a small part of the entire data. In this methodology, testing time is improved because of solving smaller sized quadratic problems and the amount of data is reduced without any significant decrease in the accuracy. We call this methodology as the Reduced Convex Hull based Image Set Distance (RCHISD) method.

Using Hypersphere Models for Image Set Classification: Another basic strategy for modeling image sets can be using hyperspheres. The geometric distance between two hyperspheres can be calculated by using only their radii and centers. When the gallery and query sets are modeled in terms of kernelized hyperspheres, this representation will be convenient due to the easiness in set-toset distance calculations. The center of the kernelized hypersphere model of the $c$-th class can be found as given in the following:

$$
\mathbf{s}_{c}=\sum_{k} \alpha_{c k}^{*} \mathbf{x}_{c k}
$$

Here, $\boldsymbol{\alpha}_{c}^{*}$ are the nonzero coefficients returned by the quadratic programming solver, and the radius of the model is given by $r_{c}=$ $\left\|\mathbf{x}_{c k}-\mathbf{s}_{c}\right\|$ for any $\mathbf{x}_{c k}$ for which $0<\alpha_{c k}^{*}<\gamma$. Finally, if $h s_{c}$ and $h s_{c^{\prime}}$ (characterized by their center and radius) are two kernelized hyperspheres, the geometric distance between them can be computed easily as:

$$
d\left(h s_{c}, h s_{c^{\prime}}\right)=\left\|\mathbf{s}_{c}-\mathbf{s}_{c^{\prime}}\right\|-\left(r_{c}+r_{c^{\prime}}\right)
$$

where

$\left\|\mathbf{s}_{c}-\mathbf{s}_{c^{\prime}}\right\|=\sqrt{\sum_{i, j} \alpha_{c i} \alpha_{c j}\left\langle\mathbf{x}_{c i}, \mathbf{x}_{c j}\right\rangle-2 \sum_{i, k} \alpha_{c i} \alpha_{c^{\prime} k}\left\langle\mathbf{x}_{c i}, \mathbf{x}_{c^{\prime} k}\right\rangle+\sum_{k, l} \alpha_{c^{\prime} k} \alpha_{c^{\prime} l}\left\langle\mathbf{x}_{c^{\prime} k}, \mathbf{x}_{c^{\prime} l}\right\rangle}$.

In this representation, using a few support vectors corresponding to the nonzero Lagrange multipliers will be enough to calculate the distances. To the best of our knowledge, this is the first time hypersphere models have been used for image set classification.

Setting Design Parameters for Reducing Image Sets: There are two design parameters in kernelized representations of the proposed methods. The first parameter is the ceiling parameter $(\gamma)$, and the accuracy is not very sensitive to it as demonstrated in the experimental work. The second parameter is the Gaussian kernel width $\sigma$, and the number of reduced samples is completely determined by $\sigma$. Using a higher value results in less number of returned samples as indicated in Fig. 4. In this example, we have demonstrated that using different values of Gaussian kernel width yields different amount of essential samples. The more number of reduced samples achieves better recognition rates but less number of reduced data yields faster testing. Since there is a trade-off between the accuracy and the real time performance, this parameter should be adjusted properly. The best parameter can result in significant improvements in storage and testing times with an inconsiderable decrease in accuracy.

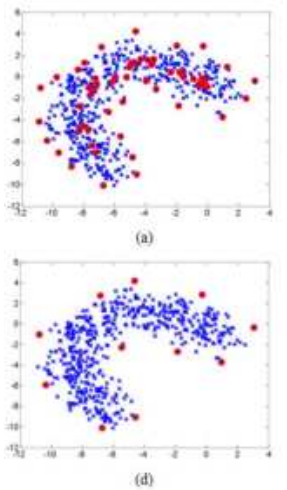

(d)
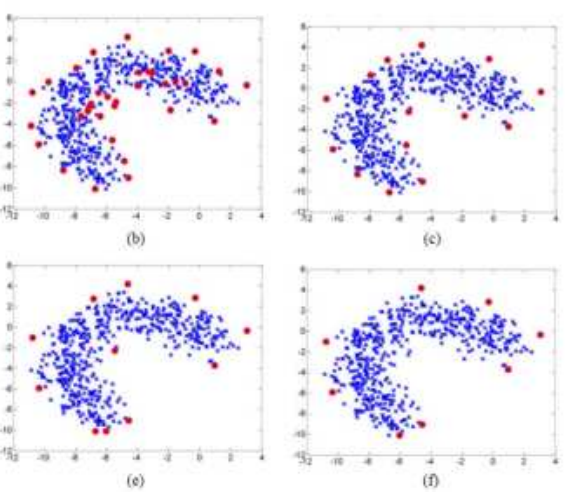

Fig. 4. An example on a toy data to demonstrate the effect of Gaussian kernel width in reduced convex hull modeling. Red circles are the support vectors returned by SVDD algorithm when the Gaussian kernel width is set to: (a) $\sigma=1.5$, (b) $\sigma=2.0$, (c) $\sigma=2.5$, (d) $\sigma=3.5$, (e) $\sigma=4.5$, (f) $\sigma=5.0$.

\subsection{Speed Improvement by Using Binary Hierarchical Decision Trees for Image Set Based Recognition}

Once we determine the essential data samples to represent image sets, we find the distances between the query set and each set in the gallery. This can be implemented in parallel since the individual set distances between a query and gallery sets are independent. In addition, we propose another approach to speed up the classification of the query set below.

When we consider individual comparisons between a query set and each gallery set, some of the comparisons may be unnecessary for a classification of a particular query. For instance, if query set belongs to a male subject, it may be further from many image sets belonging to female subjects in the gallery. Similarly, for set based general visual object classification tasks, if the query set belongs to a dog class, it is somewhat unnecessary to making comparisons between unrelated classes such as aeroplanes, cars, etc.. In order to avoid such unnecessary comparisons and to speed up the classification process, we can use a binary hierarchical decision tree (BHDT) that splits the sets in the gallery into two groups until reaching a single set for each group. In this case, one needs to make less number of comparisons between the query and the gallery; because traversing a path from the top to a bottom node takes less time for decision making as illustrated in Fig. 5. When $C$ denotes the number of person classes, this requires approximately $\log _{2} C$ comparisons in contrast to $C$ comparisons which in turn may significantly improve the testing time.

In this setup, both the accuracy and speed depend on the treestructure that creates well-balanced separable image class groups at each node of the tree. To this end, we used the Normalized Cuts 


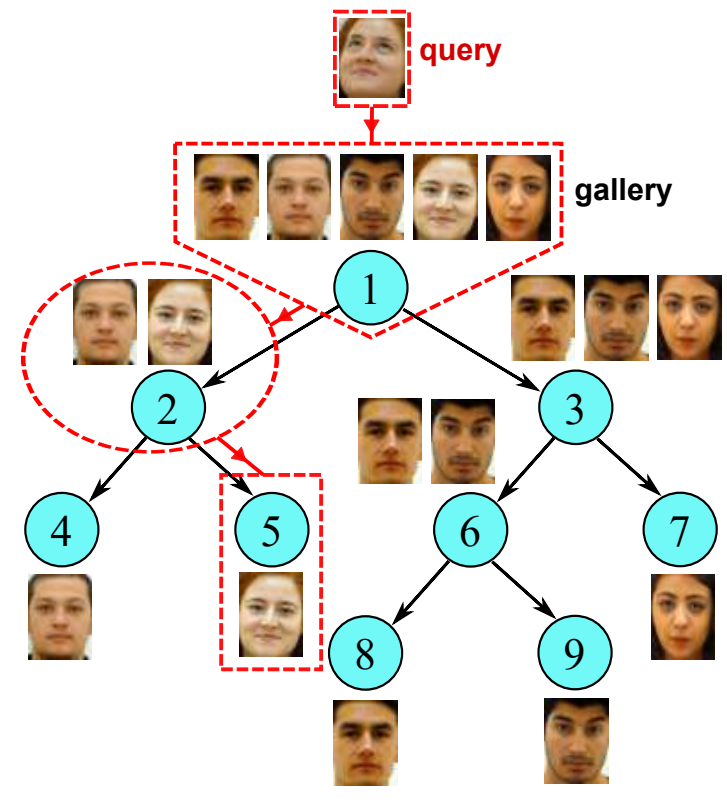

Fig. 5. A binary hierarchical decision tree (BHDT) including image sets of 5 people. BHDT includes 5 terminal and 4 internal nodes. To split the image sets into two groups in each internal node, (nodes 1,2,3 and 6), NCuts clustering algorithm is used. Query set is compared to combined sets in each internal node and assigned to the left or right node. This procedure avoids some unnecessary comparisons and improves the testing time.

(NCuts) [32] clustering to split the image sets into two groups at each node. The NCuts algorithm clusters the data into two balanced groups based on maximization of the margin between them. In order to adopt the NCuts clustering to our problem, we should consider splitting the image sets (collection of image samples) into two groups so we need to change data samples representation in the original formula with the image sets. Next, we must define a distance metric to measure the similarities between image sets to create the similarity matrix $\mathbf{W}$. Since we model each image set with a convex hull, we use the convex hull distances between the sets to measure the similarity. As a result, the edges $w_{i j}$ of the similarity matrix $\mathbf{W}$ are calculated as

$$
w_{i j}= \begin{cases}\exp \left(-d\left(H_{i}^{\text {convex }}, H_{j}^{\text {convex }}\right) / t\right), & \text { if } i \neq j \\ 0, & \text { otherwise }\end{cases}
$$

where $d\left(H_{i}^{\text {convex }}, H_{j}^{\text {convex }}\right)$ represents the distance between the convex hulls of image sets, $i$ and $j$, and $t$ is the Gaussian kernel width parameter which must be set properly. It should be noted that the size of the similarity matrix is at most $C \times C$ in our setting, where $C$ is the number of image sets in the gallery, and this size is much smaller compared to the similarity matrices returned by the classical NCuts clustering algorithm that uses the image samples rather than image sets.

Then, the image sets can be split into two groups by solving the following generalized eigenvalue problem

$$
\mathbf{L a}=\lambda \mathbf{D a}
$$

where $\mathbf{D}$ is a diagonal matrix whose elements are the columns (or rows) sums of $\mathbf{W}$ and $\mathbf{L}=\mathbf{D}-\mathbf{W}$ is the Laplacian matrix. Finally, the components of the eigenvector $\mathbf{a}^{*}$ corresponding to the second smallest eigenvalue of (18) are thresholded to split image sets into two clusters, i.e.,

$$
\begin{cases}y_{i}=-1, & \text { if } a_{i}^{*} \geq 0 \\ y_{i}=+1, & \text { if } a_{i}^{*}<0\end{cases}
$$

After splitting the image sets into two groups at each node, there are two ways to classify query sets: In the first case, we compute the kernelized convex hull distance between the query set and combined image sets in each group ${ }^{1}$. Then we assign the query set to the closest group until we reach a terminal node as illustrated in Fig. 5. The other choice might be to build nonlinear decision boundaries offline during training at each node by using gallery sets and determine the closest group of query set at each node by majority voting of individual query set samples. To this end, we train a binary nonlinear SVM classifier (recall that SVM classifier finds an hyperplane that best separates the convex hulls of two classes) separating two groups at each node, and we associate each trained classifier to the corresponding node. During testing, all query samples are classified at a particular node by using the corresponding SVM classifier in that node, and the query set is assigned to the left or right node based on the majority voting. This procedure requires only kernel function evaluations with respect to the pre-computed support vectors; hence, it is much faster compared to the first method. Therefore, we used this approach in our experiments. Both approaches require approximately $\log _{2} C$ comparisons and it improves the speed of testing time in largescale problems.

\section{EXPERIMENTS}

One can find many video based face databases for face tracking, validation, identification but just a few of them were prepared for large scale face recognition especially to be used with image sets. The most widely used video databases in set based recognition studies are namely the Honda/UCSD [33], CMU MoBo (Motion of Body) [34] and YouTube Celebrities [35] datasets. These databases are not large scale since the class size (number of people) and/or the video size (number of frames) of the databases are small. One of the few suitable larger databases for set based recognition is the COX video to video dataset [36] including 3000 video sequences of 1000 walking individuals and FaceScrub dataset [37] that includes images belonging to 530 celebrities. In this paper, we tested recognition performances and testing speeds of the proposed methods on most often used standard small sized databases and the larger sized databases COX and FaceScrub. As an alternative to these datasets, we arranged another large database consisting of 2280 videos of 285 people.

To compare different methods with the literature on different datasets, we proceed with the simple protocol given in $[3,12,4$, 14]: the detected face images were histogram equalized but no further pre-processing such as alignment or background removal was performed on them. In our database, we used 1) the gray level values, 2) the local binary pattern (LBP) features, 3) the convolutional neural network (CNN) features, and 4) OpenFace features [38] extracted from the images. In affine hull methods, we fixed the number of leading eigenvectors as the first number which satisfies to include $98 \%$ of the overall energy in eigen-decomposition

1. We run SVDD algorithm offline in order to find the most essential samples again since many pre-computed relevant samples belonging to individual image sets will no longer determine the boundary of the new combined convex hull. 
stage. In the kernelized methods we used the Gaussian kernel functions and the width parameter is determined empirically by using randomly selected subsets of each image sets. We compared the proposed method RCHISD and its fast version using BHDT (RCHISD-BHDT), to the linear/kernelized convex hull method (CHISD) [3], linear/kernelized affine hull method (AHISD) [3], SANP [11], Mutual Subspace Method (MSM) [5], Regularized Nearest Points (RNP) [12], Manifold-Manifold Distance (MMD) [4], Collaboratively Regularized Nearest Points(CRNP) [14] and Self-Regularized Nonnegative Adaptive Distance Metric Learning (SRN-ADML) [39]. In addition to these methods, we also tested linear/kernelized bounding hypersphere (HS) models for image set classification. All image sets and their extracted features used in this work as well as codes of our new methods are available at http://mlcv.ogu.edu.tr/softwares.html.

\subsection{Experiments on Small Sized Data Sets}

\subsubsection{Experiments on Honda/UCSD Data Set}

The Honda/UCSD dataset is one of the most used databases in set based recognition studies. It consists of 20 individuals and 59 video sequences with each sequence including approximately 300 500 frames. The detected faces were histogram equalized and the resulting re-sized gray scale pixel values were used as features. In the experiment, 20 sequences set aside for training are used as the gallery image sets and the remaining 39 sequences are used in testing. We did not extract LBP or CNNs for this dataset since gray level values already achieved very high accuracies.

The experimental results are given in Table 1. Here, the testing time corresponds to the time spent to classify a single test set on the average. In this experiment, the ceiling parameter of the kernelized convex hull method is changed from 0.1 to 1 , and we achieved the highest recognition accuracies (100\%) for all cases. Using reduced amount of samples did not affect the accuracy in any way and it improved testing times. RCHISD testing time is approximately 2 times faster than the traditional kernelized CHISD. Similarly, RCHISD+BHDT improves the testing time more and it is approximately 6.3 times faster than the traditional kernelized CHISD. Regarding the improvements in storage, the total number of face images in all sets was 14050 and it is reduced to 4279 in RCHISD without any drop in accuracy. In this experiment, RNP, CRNP, MMD, kernel CHISD, RCHISD, and RCHISD-BHDT methods all achieve $100 \%$ recognition accuracy. The worst performing method is the linear hyperspheres but it is the fastest method among all. The slowest methods are kernel AHISD in kernelized methods and SANP in linear methods. It should be noted that there is no big accuracy difference between the linear and kernelized methods except for the hypersphere classifier mostly because of the number of people in the dataset is small.

\subsubsection{Experiments on MoBo Data Set}

The MoBo (Motion of the Body) dataset contains 96 image sequences of 24 individuals walking on a treadmill. Images were collected from multiple cameras, and each image set includes both frontal and profile views of the subject's faces. For this database, we used LBP features from [3].

The experimental setup has been prepared the same way as given in [3]. In this setup, we randomly select one set from each class and use it in the gallery and the remaining 3 sets are used in testing. This process is repeated 10 times and we report the average
TABLE 1

Classification Rates (\%) and Testing Times on the Honda/UCSD Dataset.

\begin{tabular}{|l|c|c|}
\hline Method & Accuracy & Testing Time (sec) \\
\hline \hline Linear AHISD & 97.4 & $1.6 \mathrm{sec}$ \\
\hline Linear CHISD & 97.4 & $5.1 \mathrm{sec}$ \\
\hline Linear HS & 59.0 & $0.6 \mathrm{sec}$ \\
\hline MSM & 97.4 & $2.2 \mathrm{sec}$ \\
\hline SANP & 97.4 & $16.7 \mathrm{sec}$ \\
\hline RNP & $\mathbf{1 0 0}$ & $5.4 \mathrm{sec}$ \\
\hline CRNP & $\mathbf{1 0 0}$ & $2.6 \mathrm{sec}$ \\
\hline SRN-ADML & 97.4 & $6.2 \mathrm{sec}$ \\
\hline \hline MMD & $\mathbf{1 0 0}$ & $7.1 \mathrm{sec}$ \\
\hline Kernel AHISD & 97.4 & $14.2 \mathrm{sec}$ \\
\hline Kernel CHISD & $\mathbf{1 0 0}$ & $7.6 \mathrm{sec}$ \\
\hline Kernel HS & 94.9 & $2.8 \mathrm{sec}$ \\
\hline Kernel RCHISD & $\mathbf{1 0 0}$ & $3.7 \mathrm{sec}$ \\
\hline Kernel RCHISD-BHDT & $\mathbf{1 0 0}$ & $1.2 \mathrm{sec}$ \\
\hline
\end{tabular}

TABLE 2

Classification Rates (\%) and Testing Times on the MoBo Dataset.

\begin{tabular}{|l|c|c|}
\hline Method & Accuracy & Testing Time (sec) \\
\hline \hline Linear AHISD & $95.3 \pm 2.6$ & $32.0 \mathrm{sec}$ \\
\hline Linear CHISD & $\mathbf{9 8 . 1} \pm 0.9$ & $25.6 \mathrm{sec}$ \\
\hline Linear HS & $71.9 \pm 4.7$ & $0.6 \mathrm{sec}$ \\
\hline MSM & $92.4 \pm 1.9$ & $9.2 \mathrm{sec}$ \\
\hline SANP & $\mathbf{9 8 . 1} \pm 0.9$ & $40.2 \mathrm{sec}$ \\
\hline RNP & $93.8 \pm 2.7$ & $11.3 \mathrm{sec}$ \\
\hline CRNP & $97.4 \pm 0.8$ & $15.8 \mathrm{sec}$ \\
\hline SRN-ADML & $95.3 \pm 1.6$ & $30.0 \mathrm{sec}$ \\
\hline \hline MMD & $94.7 \pm 2.3$ & $10.6 \mathrm{sec}$ \\
\hline Kernel AHISD & $96.4 \pm 2.5$ & $87.3 \mathrm{sec}$ \\
\hline Kernel CHISD & $\mathbf{9 8 . 1} \pm 0.9$ & $32.8 \mathrm{sec}$ \\
\hline Kernel HS & $87.8 \pm 2.8$ & $5.8 \mathrm{sec}$ \\
\hline Kernel RCHISD & $97.3 \pm 1.3$ & $8.3 \mathrm{sec}$ \\
\hline Kernel RCHISD-BHDT & $95.6 \pm 1.8$ & $3.2 \mathrm{sec}$ \\
\hline
\end{tabular}

classification rates over the 10 runs. The ceiling parameter for the RCHISD method has been tested in the range $\gamma \in[0.1,1]$ and all of them returned the same accuracies. Therefore, we conclude that the results are not sensitive to this parameter so we fixed it as $\gamma=0.2$ for the rest of the experiments. Experimental results are given in Table 2.

In this experiment, the linear and kernelized convex hull models with SANP method give the best accuracies. Using RCHISD results in a $0.8 \%$ decrease in accuracy but it completes testing approximately 4 times faster than kernel CHISD and 4.8 times faster than SANP methods. Therefore reducing the image sets samples significantly improves testing times without much drop in accuracy. Similarly, RCHISD-BHDT is 2.6 times faster than RCHISD but it results in a $2.5 \%$ decrease in average recognition rate. The fastest method is the linear hypersphere method but it gives the worst recognition rate. In regard to the data reduction, RCHISD reduces the number of images in the sets from 48789 to 7098 which is a good improvement in storage. The accuracy difference between the linear and kernelized methods are not high except for the hypersphere classifier as in the previous case.

\subsubsection{Experiments on YouTube Celebrities Data Set}

The YouTube Celebrities data set contains 1910 videos of 47 celebrities that are collected from YouTube. Each sequence includes different number of frames that are mostly low resolution. 
The data set does not provide the cropped faces from videos. Therefore, we manually cropped faces using a semi-automatic annotation tool and resized them to $40 \times 40$ gray-scale images. We conduct 10 runs of experiments by randomly selecting 9 videos ( 3 for training, 6 for testing) for each experiment by following the same protocol of $[15,20]$. The averages of the classification rates and testing times are shown in Table 3.

TABLE 3

Classification Rates (\%) and Testing Times on the YouTube Celebrities Dataset.

\begin{tabular}{|l|c|c|}
\hline Method & Accuracy & Testing Time (sec) \\
\hline \hline Linear AHISD & $51.1 \pm 2.8$ & $1.2 \mathrm{sec}$ \\
\hline Linear CHISD & $57.8 \pm 2.7$ & $7.5 \mathrm{sec}$ \\
\hline Linear HS & $39.0 \pm 2.9$ & $0.8 \mathrm{sec}$ \\
\hline MSM & $50.5 \pm 3.1$ & $14.4 \mathrm{sec}$ \\
\hline SANP & $51.6 \pm 4.0$ & $39.5 \mathrm{sec}$ \\
\hline RNP & $\mathbf{6 1 . 3} \pm 2.5$ & $21.4 \mathrm{sec}$ \\
\hline CRNP & $56.7 \pm 0.5$ & $2.8 \mathrm{sec}$ \\
\hline SRN-ADML & $55.1 \pm 2.7$ & $27.8 \mathrm{sec}$ \\
\hline \hline MMD & $59.8 \pm 3.6$ & $11.9 \mathrm{sec}$ \\
\hline Kernel AHISD & $57.4 \pm 1.9$ & $24.8 \mathrm{sec}$ \\
\hline Kernel CHISD & $\mathbf{5 8 . 2} \pm 2.6$ & $25.6 \mathrm{sec}$ \\
\hline Kernel HS & $44.0 \pm 2.9$ & $1.7 \mathrm{sec}$ \\
\hline Kernel RCHISD & $57.2 \pm 2.7$ & $4.8 \mathrm{sec}$ \\
\hline Kernel RCHISD-BHDT & $55.6 \pm 2.1$ & $3.1 \mathrm{sec}$ \\
\hline
\end{tabular}

The videos in this dataset mostly include frontal views of people; therefore, linear methods perform well here. In particular, the best accuracy is obtained by RNP which is a linear method. Kernel CHISD achieves the second best result. Using reduced image sets slightly decreases the performance, but it is approximately 5.3 times faster compared to using full data. Using BHDT improves the testing time a little bit more, but the accuracy also drops around $1.6 \%$. SANP method is the worst performing method in terms of speed as in the previous cases.

\subsection{Experiments on Larger Sized Data Sets}

\subsubsection{Experiments on ESOGU-285 Face Videos Data Set}

ESOGU-285 database is a video data set which consists of 285 people with 8 videos for each person. Videos are captured in an indoor environment in two separate sessions (there are at least three weeks between the sessions) under four different scenarios. The first scenario is a kind of natural video capture; the contributors are asked to make free head movements under normal illumination conditions. The second scenario is designed to capture facial mimics and partial occlusion during talking; the contributors are asked to talk with a mobile phone in this scenario. In the third and fourth scenarios, the contributors are asked to make free head movements again but under high illumination from the left for the third, from the right for the fourth scenario. Some frames from these scenarios are shown in Fig. 6. The shortest video includes 100 frames and the longest video includes 1360 frames. The total number of the frames is 764006 in 2280 videos. This is the largest data set in terms of the total number of frames used in this study. We manually cropped the faces using a semiautomatic annotation tool such that the face images are finally re-sized to $120 \times 90$ pixels. LBP features are extracted by using $120 \times 90$ grayscale images. Grayscale features were down-sampled to $40 \times 30$ due to the memory considerations. To extract CNN features, we fine-tuned the AlexNet model of Krizhevsky et al. [40] which is trained on ILSVRC2012 dataset. We used Caffe
[41] implementation and we re-sized face images to $256 \times 256$ during fine-tuning. We set the base learning rate to $0.001 .80 \%$ of the full training data is used for training and the remaining $20 \%$ is used for validation. Iteration number is set to $180 \mathrm{~K}$. In addition to these, we also used OpenFace deep neural network [38] to extract features. In contrast to 4096-dimensional CNN features, this network outputs 128-dimensional features. We used the shared OpenFace model for this purpose.

In our experiments, we used the first session videos to form the gallery sets and the second session videos were used in testing. Experimental results are given in Table 4. CRNP method requires to operate on large sized matrices with size $n \times n$ where $n$ is the number of all frames in the gallery, so we encountered memory problem in implementation (OOM indicates the " out of memory" problem in the table). Although the SANP method is usually very slow for gray level values, it is marginally faster for LBP and CNN features. For LBP features, the best accuracy is obtained by SANP followed by kernel CHISD and MMD whereas the kernel CHISD is the best performing method alone for gray-level, CNN and OpenFace features. Kernel RCHISD method which uses the reduced amount of image sets achieves similar accuracies with kernel CHISD, but at the same time it is approximately 6 times faster for gray level features; 10 times faster for LBP, 2.8 times faster for CNN features, and 1.9 times faster for OpenFace features. In a similar manner, RCHISD-BHDT method introduces an extra improvement in testing speed with a little decrease in accuracy. The linear HS is the fastest method but again it has the worst recognition performance. In terms of storage, the total number of the frames in the database is 764006 . This size has been reduced to 149520 for gray levels; to 104716 for LBP, to 128987 for $\mathrm{CNN}$ features, to 137314 for OpenFace features by using the proposed models in the paper.

In addition to these results, we also tested a naive approach that uses AlexNet network used for CNN extraction and majority voting. To this end, we randomly selected 50 frames from each test video sequence and classified them using fine-tuned AlexNet network model. Then, we assigned the test image sequence to the class that achieves the most votes among the tested frames. This approach yielded an accuracy of $72.4 \%$ accuracy, which is slightly behind the Kernel CHISD method using CNN features. However, please note that this accuracy is significantly lower compared to the Kernel CHISD method using LBP features.

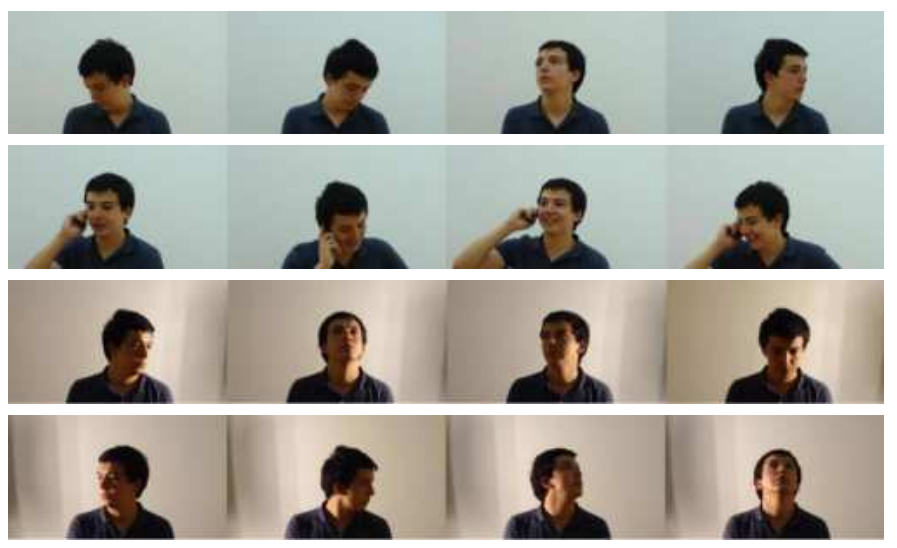

Fig. 6. Some video frames from ESOGU-285 database. Each row is selected from a different scenario numbered as 1,2,3 and 4 respectively. 
TABLE 4

Classification Rates (\%) and Testing Times on ESOGU-285 Video Dataset.

\begin{tabular}{|l|c|c|c|c|c|c|c|c|}
\hline \multirow{2}{*}{ Methods } & \multicolumn{2}{|c|}{ Grayscale Values } & \multicolumn{2}{c|}{ LBP Features } & \multicolumn{2}{c|}{ CNN Features } & \multicolumn{2}{c|}{ OpenFace Features } \\
\cline { 2 - 9 } & Accuracy & Testing Time & Accuracy & Testing Time & Accuracy & Testing Time & Accuracy & Testing Time \\
\hline Linear AHISD & 44.3 & $22.0 \mathrm{sec}$ & 66.8 & $180.0 \mathrm{sec}$ & 65.0 & $543.8 \mathrm{sec}$ & $\mathbf{6 0 . 9}$ & $2.8 \mathrm{sec}$ \\
\hline Linear CHISD & $\mathbf{5 5 . 1}$ & $179.6 \mathrm{sec}$ & 76.6 & $390.1 \mathrm{sec}$ & 65.1 & $378.6 \mathrm{sec}$ & 45.9 & $57.8 \mathrm{sec}$ \\
\hline Linear HS & 29.0 & $3.9 \mathrm{sec}$ & 39.5 & $0.8 \mathrm{sec}$ & 33.2 & $3.2 \mathrm{sec}$ & 26.3 & $0.03 \mathrm{sec}$ \\
\hline MSM & 50.1 & $2.3 \mathrm{sec}$ & 69.6 & $5.1 \mathrm{sec}$ & 63.5 & $5.6 \mathrm{sec}$ & 44.8 & $1.28 \mathrm{sec}$ \\
\hline SANP & 51.9 & $29771.0 \mathrm{sec}$ & $\mathbf{7 9 . 1}$ & $564.6 \mathrm{sec}$ & 69.4 & $1087.6 \mathrm{sec}$ & 52.2 & $7.1 \mathrm{sec}$ \\
\hline RNP & 46.7 & $1731.7 \mathrm{sec}$ & 51.9 & $2205.3 \mathrm{sec}$ & $\mathbf{7 1 . 7}$ & $367.8 \mathrm{sec}$ & 35.6 & $23.1 \mathrm{sec}$ \\
\hline CRNP & OOM & - & OOM & - & OOM & - & OOM & - \\
\hline SRN-ADML & 45.4 & $364.6 \mathrm{sec}$ & 68.4 & $380.2 \mathrm{sec}$ & 57.0 & $458.5 \mathrm{sec}$ & 31.2 & $20.2 \mathrm{sec}$ \\
\hline \hline MMD & 52.0 & $7.2 \mathrm{sec}$ & $\mathbf{7 7 . 6}$ & $30.4 \mathrm{sec}$ & 69.8 & $28.9 \mathrm{sec}$ & 52.2 & $1.8 \mathrm{sec}$ \\
\hline Kernel AHISD & 62.1 & $2015.0 \mathrm{sec}$ & 76.1 & $4369.0 \mathrm{sec}$ & 72.5 & $357.7 \mathrm{sec}$ & 71.0 & $746.4 \mathrm{sec}$ \\
\hline Kernel CHISD & $\mathbf{6 2 . 1}$ & $233.3 \mathrm{sec}$ & $\mathbf{7 7 . 6}$ & $480.4 \mathrm{sec}$ & $\mathbf{7 2 . 8}$ & $156.8 \mathrm{sec}$ & $\mathbf{7 1 . 7}$ & $84.2 \mathrm{sec}$ \\
\hline Kernel HS & 43.7 & $61.9 \mathrm{sec}$ & 49.4 & $12.9 \mathrm{sec}$ & 48.5 & $9.5 \mathrm{sec}$ & 46.4 & $7.9 \mathrm{sec}$ \\
\hline Kernel RCHISD & 61.2 & $39.7 \mathrm{sec}$ & 75.4 & $46.1 \mathrm{sec}$ & 71.7 & $55.6 \mathrm{sec}$ & 70.0 & $44.7 \mathrm{sec}$ \\
\hline Kernel RCHISD-BHDT & 60.8 & $20.6 \mathrm{sec}$ & 73.3 & $24.1 \mathrm{sec}$ & 70.2 & $25.6 \mathrm{sec}$ & 62.9 & $22.9 \mathrm{sec}$ \\
\hline
\end{tabular}

TABLE 5

Classification Rates (\%) and Testing Times on the COX Dataset.

\begin{tabular}{|l|c|c|c|c|}
\hline \multirow{2}{*}{ Methods } & \multicolumn{2}{|c|}{ Grayscale Values } & \multicolumn{2}{c|}{ LBP Features } \\
\cline { 2 - 5 } & Accuracy & Testing Time & Accuracy & Testing Time \\
\hline Linear AHISD & $42.4 \pm 7.6$ & $71.3 \mathrm{sec}$ & $44.3 \pm 9.8$ & $82.9 \mathrm{sec}$ \\
\hline Linear CHISD & $\mathbf{4 2 . 8} \pm 13.2$ & $42.9 \mathrm{sec}$ & $44.8 \pm 11.3$ & $54.3 \mathrm{sec}$ \\
\hline Linear HS & $16.5 \pm 4.2$ & $0.9 \mathrm{sec}$ & $25.1 \pm 4.9$ & $1.5 \mathrm{sec}$ \\
\hline MSM & $40.6 \pm 10.2$ & $17.3 \mathrm{sec}$ & $41.6 \pm 5.3$ & $18.6 \mathrm{sec}$ \\
\hline SANP & $41.7 \pm 10.3$ & $1856.5 \mathrm{sec}$ & $43.6 \pm 11.2$ & $978.7 \mathrm{sec}$ \\
\hline RNP & $42.5 \pm 11.3$ & $594.3 \mathrm{sec}$ & $\mathbf{4 5 . 4} \pm 13.7$ & $217.3 \mathrm{sec}$ \\
\hline CRNP & OOM & -- & OOM & -- \\
\hline SRN-ADML & $42.7 \pm 10.8$ & $354.7 \mathrm{sec}$ & $44.6 \pm 7.9$ & $351.7 \mathrm{sec}$ \\
\hline \hline MMD & $33.6 \pm 8.8$ & $42.9 \mathrm{sec}$ & $42.7 \pm 10.5$ & $60.3 \mathrm{sec}$ \\
\hline Kernel AHISD & $43.2 \pm 12.3$ & $259.9 \mathrm{sec}$ & $45.4 \pm 10.3$ & $276.4 \mathrm{sec}$ \\
\hline Kernel CHISD & $\mathbf{4 3 . 9} \pm 13.0$ & $222.9 \mathrm{sec}$ & $\mathbf{4 5 . 6} \pm 10.9$ & $250.2 \mathrm{sec}$ \\
\hline Kernel HS & $20.7 \pm 6.3$ & $4.5 \mathrm{sec}$ & $42.4 \pm 7.6$ & $71.3 \mathrm{sec}$ \\
\hline Kernel RCHISD & $41.9 \pm 11.7$ & $39.6 \mathrm{sec}$ & $44.3 \pm 11.5$ & $65.2 \mathrm{sec}$ \\
\hline Kernel RCHISD-BHDT & $40.7 \pm 13.4$ & $22.7 \mathrm{sec}$ & $41.6 \pm 10.2$ & $30.6 \mathrm{sec}$ \\
\hline
\end{tabular}

In ESOGU-285 database, there is a big difference in correct recognition rates between the linear methods and their kernelized counterparts especially for gray level values and OpenFace features in contrast to the experimental results obtained on small sized datasets. The accuracy difference between the linear and kernelized affine hull methods is $17.8 \%$ whereas the difference between linear and kernelized convex hulls is $7 \%$ for gray levels. Similarly, for OpenFace features, the accuracy difference between the linear and kernelized affine hull methods is $10.1 \%$, and the difference between linear and kernelized convex hulls is $25.8 \%$. However, for LBP features, there is still a big difference $(9.3 \%)$ between the linear and kernelized affine hull models but there is a slight difference $(1 \%)$ between the linear and kernelized convex hull models. Since convex hulls are much tighter models than the affine hulls, this result indicates that LBP features are more discriminative features compared to gray level values and they yield to more compact face manifolds. LBP features also yield to better results compared to CNNs, but the performance difference is not very significant as in gray level values. The accuracies of linear methods using OpenFace features are very low compared to the ones using CNN features, but the accuracies of nonlinear methods are similar for both $\mathrm{CNN}$ and OpenFace features. We believe that this is due to fact that CNN and OpenFace features of frontal, left and right profile views are quite different and the resulting image feature sets can be seen as kind of mixture of Gaussians where each component lie in isolated region of feature space (as opposed to a smooth nonlinear manifold). As a result, the linear models cannot approximate this structure well whereas kernel methods can successfully approximate this nonlinear model. In a similar manner, a naive approach using fine-tuned AlexNet model and majority voting yielded an accuracy lower than the proposed methods. We believe that the classical deep neural network based methods trained with single images will not work well for set based recognition where the images have different poses including full left/right profile views in addition to the frontal views. Instead, we must train such nets with image sets and enforce to minimize the distances between the different pose image features in the same set (by using Siamese or Triplet network type network structures) to obtain higher accuracies.

\subsubsection{Experiments on COX Video to Video Data Set}

The COX Faces dataset is a new dataset, which contains 3000 video sequences of 1000 walking individuals [36]. The videos are captured with three fixed camcorders when the subjects walk around the pre-designed $\mathrm{S}$-shape route. The dataset has variations in illumination, pose and resolution through this $\mathrm{S}$-shape route. For this database we used $32 \times 40$ histogram equalized face image gray-scale values and LBP features (LBP features are extracted from $32 \times 40$ face images since we do not have access to original 
TABLE 6

Classification Rates (\%) and Testing Times on the FaceScrub Dataset.

\begin{tabular}{|l|c|c|c|c|}
\hline \multirow{2}{*}{ Methods } & \multicolumn{2}{|c|}{ LBP Features } & \multicolumn{2}{c|}{ CNN Features } \\
\cline { 2 - 5 } & Accuracy & Testing Time & Accuracy & Testing Time \\
\hline Linear AHISD & $98.3 \pm 0.3$ & $2.1 \mathrm{sec}$ & $99.94 \pm 0.05$ & $6.17 \mathrm{sec}$ \\
\hline Linear CHISD & $98.2 \pm 0.3$ & $9.2 \mathrm{sec}$ & $99.97 \pm 0.04$ & $8.33 \mathrm{sec}$ \\
\hline Linear HS & $32.7 \pm 1.2$ & $0.01 \mathrm{sec}$ & $99.94 \pm 0.05$ & $0.06 \mathrm{sec}$ \\
\hline MSM & $98.1 \pm 0.3$ & $0.1 \mathrm{sec}$ & $99.94 \pm 0.05$ & $0.30 \mathrm{sec}$ \\
\hline SANP & $92.7 \pm 0.6$ & $20.5 \mathrm{sec}$ & $99.94 \pm 0.05$ & $75.40 \mathrm{sec}$ \\
\hline RNP & $\mathbf{9 9 . 6} \pm 0.1$ & $41.3 \mathrm{sec}$ & $\mathbf{1 0 0} \pm 0.00$ & $10.81 \mathrm{sec}$ \\
\hline CRNP & OOM & -- & OOM & -- \\
\hline SRN-ADML & $97.4 \pm 0.5$ & $21.7 \mathrm{sec}$ & $99.95 \pm 0.04$ & $14.78 \mathrm{sec}$ \\
\hline \hline MMD & $89.7 \pm 0.7$ & $0.5 \mathrm{sec}$ & $\mathbf{1 0 0} \pm 0.00$ & $2.45 \mathrm{sec}$ \\
\hline Kernel AHISD & $98.3 \pm 0.3$ & $12.9 \mathrm{sec}$ & $99.98 \pm 0.03$ & $76.30 \mathrm{sec}$ \\
\hline Kernel CHISD & $\mathbf{9 8 . 4} \pm 0.4$ & $13.6 \mathrm{sec}$ & $\mathbf{1 0 0} \pm 0.00$ & $42.90 \mathrm{sec}$ \\
\hline Kernel HS & $53.6 \pm 1.4$ & $2.2 \mathrm{sec}$ & $\mathbf{1 0 0} \pm 0.00$ & $2.11 \mathrm{sec}$ \\
\hline Kernel RCHISD & $97.1 \pm 0.7$ & $7.4 \mathrm{sec}$ & $\mathbf{1 0 0} \pm 0.00$ & $20.30 \mathrm{sec}$ \\
\hline Kernel RCHISD-BHDT & $95.1 \pm 0.7$ & $5.9 \mathrm{sec}$ & $99.77 \pm 0.18$ & $4.10 \mathrm{sec}$ \\
\hline
\end{tabular}

video frames) as visual features. We did not extract $\mathrm{CNN}$ features because of the small size of the face images. There are 3 image sets per person. We choose one set from each person for testing and the remaining two sets were used as gallery. For the second and the third trials, we have chosen the test set from the ones that were not used for testing earlier.

The classification rates are the averages of these three trials, and they are given in Table 5. We could not implement CRNP, because of memory issues as before since it has to operate on a large matrix. As opposed to the experiments on ESOGU faces, there are not significant differences between accuracies obtained by gray-scale values and LBP features, because LBP features are extracted from small $32 \times 40$ face images (the authors [36] share only these small cropped face images). The best classification accuracies are obtained by linear and kernelized CHISD methods. Using reduced convex hulls decreases the performance by around $2 \%$, but the speed is 5.6 times faster compared to using full data for gray-scale values. Similarly, using reduced convex hulls decreases the performance by $1.3 \%$, but the speed is 3.8 times faster for LBP features. The kernelized RCHISD method using BHDT is approximately 9.8 times faster compared to using full image sets for gray-scale values and 8.2 times faster for LBP features. SANP is again the slowest method. The total number of face image frames is 412415 and it is decreased to 41461 for gray-scale values and to 50976 for LBP features by using the proposed reduction method.

\subsubsection{Experiments on FaceScrub Data Set}

FaceScrub dataset [37] includes face images of 530 celebrities. It has been created by detecting faces based on automated search of public figures on the internet followed by manually checking and cleaning the results. In the dataset, there are 265 male and 265 female celebrities' face images whose internet links and face coordinates are given in a text file. The file includes over 100,000 face image links but some links belong to forbidden or not found web pages. First, we downloaded the dataset and cropped the faces by using the information in the supplied text file. Then, we manually checked the face images and cleaned non-face images since there were still some annotation mistakes. As a result, we had 67,437 face images of 530 celebrities with an average of 127 images (minimum 39, maximum 201) per person which is suitable to form image sets. The face images are mostly high resolution frontal face images and we resized them to $128 \times 128$. Some of the images are given in Fig. 7. Similar to the other experiments, we extracted LBP and CNN features of images.

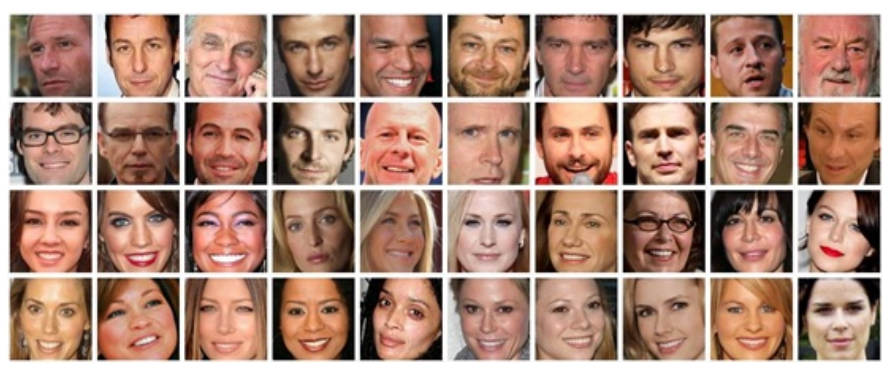

Fig. 7. Some face image samples from FaceScrub Dataset.

In our tests, we first divided the dataset into 4 equal folds, and we used the images of one fold as gallery and the remaining images are used for testing (i.e., 530 image sets are used as gallery set and the remaining $3 \times 530=1590$ image sets are used as test set). This is repeated 4 times for each fold and the final accuracy is the average of the results obtained in each trial. The results are given in Table 6. As can be seen in the results, majority of the tested methods achieve very high accuracies owing the fact that the images were mostly frontal and high quality images. As opposed to ESOGU experiments, CNN features yield higher accuracies than LBP features. It should be noted that even the worst performing linear hyperspheres method among all tested methods also achieves a very high accuracy for CNN features. This clearly shows that $\mathrm{CNN}$ features of all images in the same sets are very compact for this dataset and it can be easily modeled with simple models like a linear hypersphere. For LBP features, the best accuracies are obtained by RNP among linear methods and by Kernel CHISD method among nonlinear methods. For CNN features almost all tested methods achieve very high accuracies close to $100 \%$. The proposed reduction method, Kernel RCHISD, is 1.8 times faster compared to Kernel CHISD, and it is approximately 2.1 times faster for $\mathrm{CNN}$ features. The proposed method using binary hierarchical trees improves the testing time even more as in the other experiments. In terms of data reduction, the proposed reduction method decreases the total number of images in the entire dataset, 67437, to 35623 for LBP features and to 28272 for $\mathrm{CNN}$ features with only a minor drop in the classification 
accuracy.

\section{CONCLUSION}

In this work, we developed image set based classification methods which use the reduced amount of image samples in each set to lessen the required disk space and speed up the testing process for large scale face recognition systems. To this end, image sets are approximated with kernelized convex hulls and the set boundaries are determined based on the compact hypersphere returned by SVDD method. We call the new representation as the reduced convex hull based image set distance. The method inherently reduces the required storage, because only the samples that form the image set boundaries were kept as the essential data and there is no need to store the rest of the samples of the same set. Experimental results verify that reducing image set samples via SVDD also greatly improves the testing time without a significant drop in accuracy.

Beyond the proposed methods, we also investigated the hypersphere models to approximate the image sets. Experiments showed that hypersphere models provide extremely faster classification but they result in much lower recognition rates compared to affine hull or convex hull models. Therefore, it is not wise to use hypersphere models in final decision but they can be appropriately used to return some nearest approximate candidates from the gallery set rapidly, then a more efficient method can be used to classify the returned candidates.

Lastly, it should be noted that there is an obvious difference in recognition rates of the linear methods and their kernelized counterparts between the applications of them in small and large sized data sets. The accuracies of linear methods used for image set representations drop as the number of classes is increased. Especially, when looser linear models (such as affine hulls or linear subspaces) are used to represent the image sets, a serious drop has been observed. In this work, we also verified that some recently proposed methods reporting high recognition accuracies $[11,14]$ cannot be applied to a large sized data (even to moderate size data like our dataset) because of their slow testing speed or memory problems.

\section{ACKNOWLEDGMENTS}

This work was supported by the Scientific and Technological Research Council of Turkey (TUBITAK) with Grant number TUBITAK-114E014.

\section{REFERENCES}

[1] O. Arandjelovic, G. Shakhnarovich, J. Fisher, R. Cipolla, T. Darrell, Face Recognition with Image Sets Using Manifold Density Divergence, IEEE Computer Society Conference on Computer Vision and Pattern Recognition, 2005.

[2] Gregory Shakhnarovich, John W. Fisher, Trevor Darrell, Face Recognition from Long-Term Observations, European Conference on Computer Vision, 2002.

[3] H. Cevikalp, B. Triggs, Face recognition based on image sets, IEEE Computer Society Conference on Computer Vision and Pattern Recognition, 2010.

[4] R. Wang, S. Shan, X. Chen, W. Gao, Manifold-Manifold Distance with application to face recognition based on image set, IEEE Computer Society Conference on Computer Vision and Pattern Recognition, 2008.
[5] O. Yamaguchi, E. Fukui, K. Maeda, Face recognition using temporal image sequence, IEEE International Conference on Automatic Face and Gesture Recognition, 1998.

[6] J. Hamm, D. D. Lee, Grassmann Discriminant Analysis: A Unifying View on Subspace-based Learning, International Conference on Machine Learning, 2008.

[7] T. Wang, P. Shi, Kernel Grassmannian Distances and Discriminant Analysis for Face Recognition from Image Sets, Pattern Recognition Letters, vol. 30, pp. 1161-1165, 2009.

[8] M. T. Harandi, C. Sanderson, S. Shirazi, B. C. Lovell, Graph Embedding Discriminant Analysis on Grassmannian Manifolds for Improved Image Set Matching, IEEE Computer Society Conference on Computer Vision and Pattern Recognition, 2011.

[9] Z. Huang, R. Wang, S. Shan, X. Li, X. Chen, Log-euclidean Metric Learning on Symmetric Positive Definite Manifold with Application to Image Set Classification, International Conference on Machine Learning, 2015.

[10] Z. Huang, R. Wang, X. Li, W. Liu, S. Shan, L. Van Gool, $\mathrm{X}$. Chen, Geometry-Aware Similarity Learning on SPD Manifolds for Visual Recognition, IEEE Transactions on Circuits and Systems for Video Technology, vol. 28, pp. 2513-2523, 2018.

[11] Y. Hu, A. S. Mian, R. Owens, Face Recognition Using Sparse Approximated Nearest Points between Image Sets, IEEE Transactions on Pattern Analysis and Machine Intelligence, vol. 34, pp. 1992-2004, 2012.

[12] M. Yang, P. Zhu, L. Van Gool, L. Zhang, Face recognition based on regularized nearest points between image sets, IEEE International Conference and Workshops on Automatic Face and Gesture Recognition, 2013.

[13] W. Wang, R. Wang, S. Shan, X. Chen, Prototype Discriminative Learning for Image Set Classification, IEEE Signal Processing Letters, vol. 24, pp. 1318-1322, 2017.

[14] Y. Wu, M. Minoh, M. Mukunoki, Collaboratively Regularized Nearest Points for Set Based Recognition, British Machine Vision Conference, 2013.

[15] P. Zhu, W. Zuo, L. Zhang, S. C. K. Shiu, D. Zhang, Image Set-Based Collaborative Representation for Face Recognition, IEEE Transactions on Information Forensics and Security, vol. 9, pp. 1120-1132, 2014.

[16] Z. Cui, H. Chang, S. Shan, B. Ma, X. Chen, Joint sparse representation for video-based face recognition, Neurocomputing, vol. 135, pp. 306-312, 2014.

[17] Y.-C. Chen, V. M. Patel, S. Shekhar, R. Chellappa, P. J. Phillips, Video-based face recognition via joint sparse representation, IEEE International Conference and Workshops on Automatic Face and Gesture Recognition, 2013.

[18] S. Chen, C. Sanderson, M. T. Harandi, B. C. Lovell, Improved Image Set Classification via Joint Sparse Approximated Nearest Subspaces, IEEE Computer Society Conference on Computer Vision and Pattern Recognition, 2013.

[19] W. Fan, D.-Y. Yeung, Locally Linear Models on Face Appearance Manifolds with Application to Dual-Subspace Based Classification, IEEE Computer Society Conference on Computer Vision and Pattern Recognition, 2006.

[20] R. Wang, X. Chen, Manifold Discriminant Analysis, IEEE Computer Society Conference on Computer Vision and Pattern Recognition, 2009.

[21] A. Hadid, M. Pietikainenn, From still image to video-based face recognition: an experimental analysis, IEEE International 
Conference on Automatic Face and Gesture Recognition, 2004.

[22] M. Hayat, M. Bennamoun, S. An, Deep Reconstruction Models for Image Set Classification, IEEE Transactions on Pattern Analysis and Machine Intelligence, vol. 37, pp. 713$727,2015$.

[23] M. Hayat, M. Bennamoun, S. An, Learning Non-linear Reconstruction Models for Image Set Classification, IEEE onference on Computer Vision and Pattern Recognition, 2014.

[24] Y. Liu, J. Yan, W. Ouyang, Quality Aware Network for Set to Set Recognition, IEEE Conference on Computer Vision and Pattern Recognition, 2017.

[25] J. Yang, P. Ren, D. Zhang, D. Chen, F. Wen, H. Li, G. Hua, Neural Aggregation Network for Video Face Recognition, IEEE Conference on Computer Vision and Pattern Recognition, 2017.

[26] Y. Rao, J. Lin, J. Lu, J. Zhou, Learning Discriminative Aggregation Network for Video-Based Face Recognition, International Conference on Computer Vision, 2017.

[27] L. Liu, L. Zhang, H. Liu, S. Yan, Toward Large-Population Face Identification in Unconstrained Videos, IEEE Transactions on Circuits and Systems for Video Technology, vol. 24, pp. 1874-1884, 2014.

[28] D. M. Tax and R. P. Duin, Support Vector Data Description, Machine Learning, vol. 54, pp. 45-66, 2004.

[29] M. Yalcin, H. Cevikalp, H. S. Yavuz, Towards Large-Scale Face Recognition Based on Videos, IEEE International Conference on Computer Vision Workshops, 2015.

[30] K. P. Bennett, E. J. Bredensteiner, Duality and Geometry in SVM Classifiers, International Conference on Machine Learning, 2000.

[31] B. Schölkopf, J. C. Platt, J. Shawe-Taylor, A. J. Smola, R. C. Williamson, Estimating the Support of a High-Dimensional Distribution, Neural Computation, vol. 13, pp. 1443-1471, 2001.

[32] J. Shi, J. Malik, Normalized cuts and image segmentation, IEEE Transactions on Pattern Analysis and Machine Intelligence, vol. 22, pp. 888-905, 2000.

[33] K.-C. Lee, J. Ho, M.-H. Yang, D. Kriegman, Visual tracking and recognition using probabilistic appearance manifolds, Computer Vision and Image Understanding, vol. 99, pp. 303331, 2005.

[34] R. Gross, J. Shi, The CMU Motion of Body (MoBo) Database, Carnegie Mellon University, Pittsburgh, PA, Tech. Rep. CMU-RI-TR-01-18, 2001.

[35] M. Kim, S. Kumar, V. Pavlovic, H. Rowley, Face tracking and recognition with visual constraints in real-world videos, IEEE Conference on Computer Vision and Pattern Recognition, 2008.

[36] Z. Huang, S. Shan, R. Wang, H. Zhang, S. Lao, A. Kuerban, X. Chen, A Benchmark and Comparative Study of VideoBased Face Recognition on COX Face Database, IEEE Transactions on Image Processing, vol. 24, pp. 5967-5981, 2015.

[37] H.-W. Ng, S. Winkler, A data-driven approach to cleaning large face datasets, IEEE International Conference on Image Processing, 2014.

[38] B. Amos, B. Ludwiczuk, M. Satyanarayanan, OpenFace: A general-purpose face recognition library with mobile applications, CMU School of Computer Science, Tech. Rep. CMUCS-16-118, 2016.

[39] A. Mian, Y. Hu, R. Hartley, R. Owens, Image Set Based Face
Recognition Using Self-Regularized Non-Negative Coding and Adaptive Distance Metric Learning, IEEE Transactions on Image Processing, vol. 22, pp. 5252-5261, 2013.

[40] A. Krizhevsky, I. Sutskever, G. E. Hinton, ImageNet Classification with Deep Convolutional Neural Networks, International Conference on Neural Information Processing Systems, 2012.

[41] Y. Jia, E. Shelhamer, J. Donahue, S. Karayev, J. Long, R. Girshick, S. Guadarrama, T. Darrell, Caffe: Convolutional Architecture for Fast Feature Embedding, ACM International Conference on Multimedia, 2014.

[42] S. A. Nene, S. K. Nayar, H. Murase, Columbia Object Image Library (COIL-100), Columbia University, Computer Science Department, New York, USA, Tech. Rep. CUCS-00696, 2016.

[43] B. Schölkopf, A. Smola, K.-R. Müller, Kernel principal component analysis, Advances in Kernel Methods - Support Vector Learning, pp. 327-352, 1999.

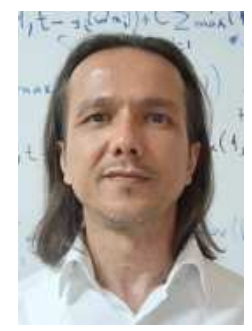

Hakan Cevikalp received his M.S. degree from the Department of Electrical and Electronics Engineering, Eskisehir Osmangazi University, Eskisehir, Turkey, in 2001 and a Ph.D. degree from the Department of Electrical Engineering and Computer Science, Vanderbilt University, Nashville, TN, USA in 2005. He is currently a full professor in Electrical and Electronics Engineering Department of Eskisehir Osmangazi University. His research interests include matimization, and computer vision. chine learning, image and signal processing, op-

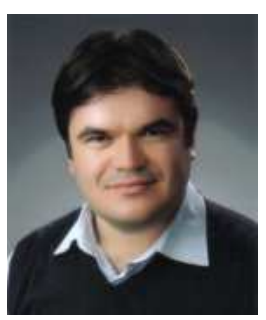

Hasan Serhan Yavuz received B.S., M.S. and $\mathrm{PhD}$ degrees from the Electrical and Electronics Engineering Department of Eskisehir Osmangazi University, Eskisehir, Turkey, in 1999, 2002 , and 2008 respectively. He is currently in the academic staff of the same department as an assistant professor. His research interests include image processing, fuzzy logic, computer vision and pattern recognition.

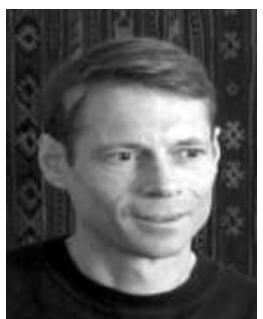

Bill Triggs is a CNRS researcher who works mainly on machine learning based approaches to understand images and other sensed data. $\mathrm{He}$ leads the Al (Apprentissage et Interfaces) team in the Laboratoire Jean Kuntzmann (LJK) in Grenoble, France, and he is also the deputy director of LJK, coordinator of the EU research project CLASS on unsupervised image and text understanding, and coordinator of the CNRS partner of the EU network of excellence PASCAL 2. 